\title{
Structure and Dynamics in Biology at the Nanoscale: X-ray Tools
}

\author{
Greg L Hura*\#
}

*Lawrence Berkeley National Laboratory, 1 Cyclotron Rd. Berkeley CA 94720 USA

\#Department of Chemistry and Biochemistry, University of California at Santa Cruz, Santa Cruz CA 95064 USA

Biological macromolecules function by adopting conformationally-distinct states through processes such as phosphorylation, nucleotide binding, ATP hydrolysis, ligand binding, complex formation, or combinatorial post-translational modification. Many of these conformational states may be biologically important, but relatively few are observed and compared by current structural techniques. Crystallography is precise but low throughput, typically captures one low-energy conformation, and often requires truncations or mutations. SAXS experiments can probe the solution state under any condition, provide information at resolutions sufficient to distinguish conformational states, characterize flexible macromolecules and screen in high throughput. Moreover SAXS can be accurately calculated from atomic resolution models forming a valuable complementarity between crystallography and SAXS. Here we present several examples of the application of SAXS which significantly enhance our understanding of biological networks beyond what either a high resolution structure or informatics analysis can yield.

For macromolecular engineering the same combinatorial challenges exist. Because our understanding of the chemical and structural implications of even one point mutation is limited, techniques are required with rapid throughput to build intuition. Moreover crystallography, typically provides perspective in one stochastic solvent condition whereas engineered molecules may be designed for many solvent environments. A crystal structure of one construct combined with computational modelling and SAXS data on mutations and in varying solution conditions can provides remarkable insights for engineering. We will describe experimental and analytical frameworks that have aided in engineering projects with applications in human health and commercial chemical synthesis. 